\title{
Erratum to: Optimal Channel Sensing Order for Various Applications in Cognitive Radio Networks
}

\author{
Junyuan Huang • Huaibei Zhou • Yongqun Chen • \\ Bo Chen · Xingyu Zhu • Ruoshan Kong
}

Published online: 27 November 2012

C Springer Science+Business Media New York 2012

\section{Erratum to: Wireless Pers Commun \\ DOI 10.1007/s11277-012-0906-x}

There is an error in the Acknowledgments section of the original publication. The grant number specified for the support by the National 973 Project of China should be No. 2009CB320400 (not 2009CB320402).

The online version of the original article can be found under doi:10.1007/s11277-012-0906-x.

J. Huang $(\varangle) \cdot$ Y. Chen $\cdot$ B. Chen $\cdot$ X. Zhu

School of Physics and Technology, Wuhan University, Wuhan 430072, China

e-mail: leon.hjy@gmail.com

Y. Chen

e-mail: yiyuxiniao@gmail.com

B. Chen

e-mail: innerpeacer@gmail.com

X. Zhu

e-mail: zhuxingyu_whu@163.com

H. Zhou $\cdot$ R. Kong

International School of Software, Wuhan University, Wuhan 430072, China

e-mail: bzhou@whu.edu.cn

R. Kong

e-mail: krs1024@126.com 